\title{
Una escena truncada: historia de la Escuela de Teatro de la Universidad Austral de Chile (1970-1976)*
}

\author{
Sibila Sotomayor, U. de Valparaíso / U. Catholique de Louvain \\ sibila.vanr@gmail.com
}

\author{
Angélica San Martín, U. de Valparaíso / U. de Buenos Aires \\ angelicapazsanmarting@gmail.com
}

Resumen

Si bien tradicionalmente se asume que las únicas universidades que impartieron la formación de actor profesional antes de 1990 fueron la Universidad de Chile, la Pontifica Universidad Católica y la Universidad de Chile sede Valparaíso, existió una cuarta institución, la Universidad Austral de Chile, que contaba con una malla curricular y programas apropiados, en un intento por reforzar la profesionalización actoral en el área sur de nuestro país. En este artículo presentamos los resultados de nuestra memoria de grado respecto de este trozo olvidado de la historia teatral chilena, el que nos permite dimensionar la contundencia del movimiento teatral a nivel nacional tanto a lo largo de la historia como en la actualidad.

Palabras clave

Teatro universitario, historia del teatro, teatro regional, teatro chileno, Valdivia.

\section{A truncated scene: history of the School of Theater of the Universidad Austral de Chile (1970-1976)}

\begin{abstract}
While it is traditionally assumed that the Universidad of Chile, the Pontificia Universidad Católica de Chile, and the Universidad de Chile's Valparaíso campus were the only emblematic universities with degrees in professional acting before 1990, there was indeed a fourth establishment that also offered this major: The Universidad Austral de Chile. With relevant curriculum and programs, this university attempted to strengthen the professionalization of actors in the southern area of our country. This article pre-sents results from our undergraduate thesis, which aimed to recover an overlooked portion of Chilean theater history. In doing so, we were able to assess the strength of the theater movement on a national level, both historically and currently.
\end{abstract}

Keywords

University theatre, history of theatre, regional theatre, Chilean theatre, Valdivia.

* Recibido: 15 de marzo de 2016 / Aceptado: 29 de mayo de 2016. 


\section{Introducción}

Desde la apertura de escuelas de formación artística teatral en Chile en la década de los 1940, con la fundación del Teatro Experimental de la Universidad de Chile y del Teatro de Ensayo de la Universidad Católica, se incorporó el oficio del actor como una carrera que debía ser impartida por una institución universitaria. Ambas escuelas generaron un precedente de modelo educativo universitario de formación actoral en nuestro país que, en la década de los 1960, sujeto a la reforma universitaria, intentaría ser replicado fuera de Santiago (Hurtado, 2003). En ese contexto fue que surgieron la Escuela Universitaria de Teatro sede Valparaíso de la Universidad de Chile y la Escuela de Teatro de la Universidad Austral de Chile, en Valdivia. La primera es antecesora de la actual Escuela de Teatro de la Universidad de Valparaíso, institución en la que cursamos nuestros estudios superiores y donde realizamos, por ende, la memoria de grado que originó este artículo (San Martín \& Sotomayor, 2012). No obstante, es la segunda la que concita nuestra atención en el presente estudio.

Los procesos de profesionalización del arte del actor son investigados con interés, al repercutir sobre la configuración del campo teatral y, desde luego, sobre la concepción misma del teatro y su práctica. En esta línea de pesquisas, concentrarse en lo ocurrido en una ciudad capital como es Santiago de Chile ha sido un paso necesario, pero que requiere completarse con una mirada hacia los procesos regionales, especialmente si lo es la experiencia teatral propia. De allí la relevancia de detenerse una escuela de teatro como es la de la Universidad Austral de Chile. En efecto, si bien esta escuela es influida por el modelo teatral universitario santiaguino, muestra al respecto algunas diferencias significativas que contribuyen a entender su accidentada trayectoria en el difícil contexto histórico, político y social de los años 1960 a 1980. Así, con la finalidad de aportar a la historia del teatro universitario nacional, buscamos recuperar la evolución de la Escuela de Teatro de la Universidad Austral de Chile desde su fundación hasta su cierre, el año 1976.

Los antecedentes sobre esta escuela son prácticamente inexistentes, viviendo solamente en los recuerdos de aquellos que fueron parte de su historia. Por ello, tras revisar tales antecedentes y sus variantes, nuestro estudio de historia teatral se basó en dos tipos de fuentes. Por un lado, trabajamos con fuentes obtenidas mediante levantamiento hemerográfico y análisis de material crítico de prensa; levantamiento bibliográfico de notas de actas, decretos universitarios, bibliografía temática y archivos de y sobre la Escuela de Teatro de la Universidad Austral de Chile. Por otro lado, recabamos datos a través de entrevistas en profundidad a personas claves asociadas a esta historia, las que, debido a su experiencia personal, podían entregarnos datos concretos al respecto. Es el caso en especial de Consuelo Holzapfel, Roberto Matamala, Juan Ossa y Margarita Poseck, así como 
del maestro Juan Barattini, quien nos entregó información complementaria sobre el teatro universitario. De esta manera, junto a las fuentes escritas con informaciones directas o indirectas sobre la institución, trabajamos con la memoria y el testimonio de quienes participaron en ella.

\section{Primeros teatros universitarios chilenos}

Previo a la fundación de los teatros universitarios, el teatro en Chile vivía un proceso de enriquecimiento cultural gracias a los aportes e influencias de artistas y compañías extranjeras (Pradenas, 2006: 222-223). Como consecuencia de la Guerra Civil Española y de la Segunda Guerra Mundial, muchos artistas consagrados debieron dejar sus países de origen por motivos políticos, lo que generó las condiciones propicias para que se concretara la renovación del teatro y del arte en Chile (Hurtado, 2010: 16). Estas influencias fueron de gran importancia, pues impulsaron el proceso de profesionalización universitaria del teatro chileno. Sin embargo, faltaba la posibilidad de adquirir este oficio no sólo a través de la experiencia y la enseñanza de grandes maestros, sino a través de una institución respaldada por un sistema educacional nacional. La aparición de los teatros universitarios era necesaria para respaldar la profesionalización total del oficio del actor (Hurtado, 2003).

La fundación del Teatro Experimental en 1941 dio paso, en 1946, a la creación de la Escuela de Teatro de la Universidad de Chile. El reconocido funcionamiento de la compañía universitaria despertó el interés de las autoridades universitarias, las que decidieron formalizarla con la inclusión de este oficio como una carrera más dentro de la oferta académica. El Teatro de Ensayo de la Universidad Católica, por su parte, fue fundado en 1943, mientras que la respectiva Escuela de Teatro lo fue en 1947 (Revista Apuntes, 2003). Ambas escuelas fueron el punto de partida de la historia formativa teatral universitaria en Chile.

En relación a los modelos de formación teatral extranjeros, el chileno se diferenció por surgir en universidades. Debido a que tanto la educación como las artes en general cumplían un importante rol en el desarrollo de nuestro país, siendo promovidos por los gobiernos de la época en especial mediante las actividades de extensión universitaria, es coherente que el modelo chileno se haya originado de esta forma (Hurtado, 2010: 16). Los teatros universitarios marcaron un hito en la historia del teatro chileno, pues establecieron un modelo educacional universitario para la formación del actor que llega hasta nuestros días y que, por lo demás, sólo se puede encontrar de manera similar en México hoy. Por ello, los teatros universitarios no sólo marcaron el teatro nacional, sino que también denotaron una diferencia respecto a la formación teatral en otros países, tanto latinoamericanos como europeos y norteamericanos (Hurtado \& Barría, 2010: 19). 
Años más tarde, la formación artística teatral universitaria se expandió hacia otras ciudades, potenciando la descentralización de las compañías de teatro universitarias y la creación de escuelas de teatro en diversas regiones a lo largo del país (Zegers, 1999: 25). Todas estas iniciativas se vieron favorecidas debido al contexto político social en que surgieron. La aparición de los teatros universitarios, notablemente, coincidió con un apogeo en el fortalecimiento de la educación pública (Sotoconil, 1991: 68), mientras que la expansión de escuelas de formación hacia regiones coincidió con la Reforma Universitaria. En este último periodo, se generó asimismo un cambio de enfoque respecto a la producción artística de los teatros universitarios, comenzando a cuestionarse las antiguas tendencias en la producción teatral (Ochsenius, 1982: 126).

La preocupación por vincular la actividad teatral con el contexto de transformaciones sociales que vivía el país fue propicia a la realización de cambios en la selección de repertorios y en las metodologías de creación, orientándose estas últimas hacia la interrelación entre actores, directores y dramaturgos, con el fin de potenciar la creación colectiva. A través de estos cambios, se pretendía dirigir el teatro hacia nuevas audiencias y en particular, hacia los sectores populares (Ochsenius, 1982: 127).

Lo anterior se vio favorecido por el gobierno de Eduardo Frei Montalva y potenciado por el de la Unidad Popular (U.P.), encabezado por Salvador Allende Gossens. La vía chilena al socialismo significaba una reestructuración de la sociedad, pues pretendía modificar el modelo socioeconómico que regía al país, encaminándolo hacia la igualdad. Además, la U.P. había llegado al poder a través de la vía democrática, lo que suponía un avance mayor tanto para el socialismo como para la nación en su totalidad y para la historia internacional, considerando el contexto de Guerra Fría en que se encontraba el mundo (Moulian, 1997: 158). Sin embargo, como se sabe, tras un efímero período de tres años, el gobierno de la U.P. llegó a su fin, debido al golpe de Estado que las Fuerzas Armadas del país llevaron a cabo el día 11 de septiembre de 1973.

Este quiebre político tuvo un impacto mayor en la historia nacional, significando, en el ámbito que nos interesa, que el desarrollo del teatro universitario, continuo durante treinta años, sufriera un retroceso y un estancamiento que duró más de dos décadas. En efecto, este desarrollo se vio potenciado o, al contrario, perjudicado por los diversos enfoques de los gobiernos del momento, a pesar de no contar con una motivación directa por parte de ellos. En Santiago y Valparaíso, las escuelas de teatro universitario permanecieron o fueron restablecidas años después. En Valdivia, sin embargo, la escuela de teatro de la UACH fue el último antecedente de formación teatral profesional de la región. Con la imposición del 
Gobierno Militar, las iniciativas descentralizadoras en el teatro universitario se eliminaron por completo y algunas escuelas, simplemente, desaparecieron. Entre ellas, la Escuela de Teatro de la Universidad Austral de Chile.

\section{Creación de la Escuela de Teatro de la Universidad Austral de Chile}

Debido a la preocupación de los fundadores de la Universidad Austral de Chile ${ }^{1}$ respecto al desarrollo de las artes y de la cultura en la ciudad de Valdivia, es que una de las facultades originales de esta casa de estudios fue la Facultad de Bellas Artes (Almonacid Zapata, 2004: 189). La Universidad comenzó a funcionar en el año 1955 con una matrícula de 270 alumnos, estando conformada por la Escuela de Artes Plásticas, el Ballet Folklórico, el Conservatorio de Música y el Coro Universitario.

Tras múltiples crisis, un receso y diversas reestructuraciones que evidencian la poca capacidad de generar una estructura orgánica sustentable, la Facultad de Bellas Artes de la UACH logra consolidarse con una renovada planta académica adscrita al nuevo Instituto de Artes Plásticas. La impronta del Instituto se signó en la experimentación artística, acompañada de una profunda reflexión teórica e interpretativa. De este modo, el Instituto de Artes Plásticas fue el centro neurálgico de una nueva generación de creadores que se erigieron como destacados actores sociales y culturales, elevando cualitativamente la práctica artística realizada en la ciudad. Además, en 1967, se crea el curso de Pedagogía en Música y, en 1970, en una sede de la Facultad de Bellas Artes ubicada en la calle General Lagos № $1107^{2}$, se crea la Escuela de Teatro, tema central de nuestra investigación (Almonacid Zapata, 2004: 211).

La fundación de la Escuela de Teatro de la UACH se relaciona directamente con el actor, profesor, director e investigador Rubén Sotoconil. Al haber cumplido un

\footnotetext{
1 Tras una gestión de más de una década encabezada por el Dr. Eduardo Morales Miranda, la Sociedad Amigos del Arte (SAA) y otras personalidades locales valdivianas, la Universidad Austral de Chile fue fundada el 7 de septiembre de 1954, a partir del Decreto Supremo № 3.757, bajo el lema de Libertas Capitur, siendo inaugurada el 12 de marzo de 1955. A pesar de no ser una universidad propia del Estado, en sus primeros 14 años de funcionamiento se encontró bajo la tutela examinadora de la Universidad de Chile, como consecuencia del Estatuto Orgánico de la Enseñanza Universitaria vigente en el país en ese entonces. Por ello, debía regirse por parámetros establecidos por la sede central de la Universidad de Chile en Santiago. (Almonacid Zapata, 2004: 195). Sin embargo, y tras un largo proceso jurídico, la UACH obtuvo su total autonomía el 3 de junio de 1968, gracias a la promulgación de la Ley 16.848 (Almonacid Zapata, 2004: 221).

2 En donde, actualmente, se encuentra el Conservatorio de Música de la misma casa de estudios.
} 
rol como fundador del Teatro Experimental de la Universidad de Chile en Santiago, poseía la experiencia necesaria para intentar replicar un modelo de formación teatral universitario en la ciudad de Valdivia. Su finalidad era descentralizar la actividad y la formación teatral nacional (Ossa, 2011), sumándose así a las iniciativas generadas en el norte, el centro y el sur de nuestro país.

Sin embargo, él no pretendía sólo fundar una nueva compañía teatral universitaria, como había sido el caso en Antofagasta y Concepción. El propósito de Sotoconil en Valdivia era instalar una escuela de teatro de nivel profesional y universitario que le entregara a la comunidad regional los profesionales del área teatral que ella requería. Por medio de la Escuela de Teatro de la $\cup A C H$, se trataba de mantener activa una cartelera teatral local que era complementada con la visita constante de compañías de la capital. Sin embargo, tras el golpe militar de 1973, la Universidad Austral de Chile experimentó una nueva reorganización interna y, a pesar de sobrevivir tres años durante la dictadura, la Escuela de Teatro fue cerrada con sólo siete años de funcionamiento (1970-1976).

Dentro de la corta vida de esta Escuela, que fue la de menor duración al interior de la Facultad de Bellas Artes de la UACH, es posible distinguir dos grandes etapas bastante diferentes entre sí. La primera comprende desde su fundación en el año 1970 hasta el año 1973 y la segunda, del año 1974 al 1976. El quiebre del año 1973 trajo consigo la reestructuración interna de la Universidad y de la malla curricular de la Carrera de Teatro.

\section{Primer periodo, 1970-1973}

El primer período de la Escuela de Teatro de la Universidad Austral de Chile se caracteriza por estar regido por un enfoque particular, distinto al santiaguino. En efecto, se estimaba, en ese entonces, que la real y primera necesidad de la región en ese ámbito era la de formar Monitores teatrales que pudieran difundir y enseñar las artes escénicas en la región (Universidad Austral de Chile, 1972: 2 ). Por ello, se creó la Carrera de Monitor Teatral y, paralelamente, se puso en funcionamiento un Taller de Teatro, denominado "Grupo Libre". Éste, que poseía una menor carga horaria que la Carrera de Monitores, era una alternativa pensada para trabajadores o estudiantes de otras carreras que poseyeran interés por el teatro. De acuerdo con estos lineamientos, el primer llamado público para formar parte de la Escuela de Teatro se realizó el viernes 7 de abril de 1970. En palabras de Sotoconil:

Como muchos elementos valiosos no pueden atender al horario de la academia (por ser alumnos de otras facultades o trabajar hasta tarde) tendré también un "grupo libre", para montar obras [...]. Se constituye un grupo de 18 a 20 en Academia, con 14 
horas de clases y otro libre, con 6 horas de ensayo. Confío en que en el camino queden menos y se puedan constituir homogéneamente (Sotoconil, 1970).

Durante ese año, el artista y profesor asumió labores en docencia, dictando la cátedra de Actuación. También ejerció la dirección del Departamento de Teatro, de la Carrera de Teatro y del Departamento de Extensión de la Universidad, firmando un contrato que lo mantuvo en el sur del país hasta febrero de 1971 (UACH, 1972: 2). Además, definió los reglamentos internos de la Escuela y realizó sus primeras presentaciones. Con muchas tareas a su haber, delegó la preparación corporal de los estudiantes a la bailarina clásica y directora de la Escuela de Ballet de la UACH, Matilde Romo, en la asignatura de Movimiento. A su vez, integró al presidente de la Asociación de Judo de Valdivia, quien además era profesor de física y matemática, Leopoldo Morandé. Él estuvo a cargo de complementar el área corporal impartiendo clases de Judo tres veces a la semana. Por otra parte, Hernán Poblete, Secretario General de la Universidad, estuvo desde sus inicios ligado a la actividad literaria en Valdivia, por lo que dictó la asignatura de Historia del Teatro.

Para la muestra final del primer semestre, la búsqueda de la obra comenzó con Lisistrata, de Aristófanes, pero fue descartada por la polémica que podría causar, dadas las explícitas alusiones sexuales que contenía la pieza. Por ello fue que, finalmente, en julio de ese año, comenzó la preparación de la obra Ánimas de día claro, de Alejandro Sieveking. Para el segundo semestre, el conjunto de Monitores montó la obra i Perdido Gracias a Dionisio!, del estudiante Roberto Matamala, quien participaba en el taller de dramaturgia, también dictado por Sotoconil. Por su lado, el Grupo Libre, conocido también como "Taller de Teatro", trabajó la creación colectiva La cáscara y la Pluma, protagonizada por el estudiante Luis "Tato" Montes. Con estos montajes, la Escuela de Monitores y su taller vespertino cerraron el primer año de la Escuela de Teatro de la Universidad Austral de Chile.

A comienzos de 1971, Rubén Sotoconil, satisfecho por la labor que había realizado durante más de un año, tomó la decisión de volver con su familia a la capital. Los factores climáticos y emocionales, sumados a la deficiente retribución económica, terminaron por prevalecer, frente a la idea de permanecer en el sur un año más. Además, en esos momentos, la Escuela de Teatro ya estaba asentada en la Facultad de Bellas Artes y, por ende, en la Universidad, por lo que estimaba que la tarea que le había sido asignada ya estaba cumplida.

Antes de partir de la ciudad de Valdivia, dejó a cargo de la Escuela a Hernán Poblete y se contactó con Juan Guzmán Améstica quien, posteriormente, con- 
tactó a Jaime Silva. Estos dramaturgos, ya ligados a la ciudad de Valdivia, formarían parte de la nueva directiva de la Escuela de Teatro³. En 1971, Juan Guzmán Améstica asumió la dirección de la Escuela de Teatro, mientras que el Departamento de Teatro quedó a cargo de Hernán Poblete. Por su parte, el área de Extensión mantuvo la promoción de la actividad teatral en colegios, sindicatos y centros culturales tanto en Valdivia como en otras localidades de la provincia.

En ese mismo año, la Facultad de Bellas Artes de la UACH efectuó un nuevo llamado abierto al público, en el periódico El Correo de Valdivia, para captar la atención de interesados en participar tanto en la Escuela de Monitores como en el Taller de Teatro. Los requisitos para ser alumno regular de la Carrera de Monitores eran: ser mayor de 16 años con licencia secundaria; rendir el examen de admisión o prueba especial; acreditar con certificado médico que el postulante tenía una salud compatible con los estudios; y, por último, cancelar el valor de la matricula establecida por la Universidad. Tanto la Carrera de Monitor Teatral como el Grupo Libre de Teatro eran de carácter vespertino. La Carrera de Monitores atendía de lunes a viernes de 18:30 a 22:00 horas y el Grupo Libre, que tenía un horario más reducido, los martes, miércoles y jueves de 20:00 a 22:00 hrs.

Las pruebas especiales de ingreso se dividían en dos etapas: la primera de ellas consistía en analizar un texto en una improvisación grupal y preparar, durante tres días, una escena de una obra escogida por los académicos. Luego, se realizaba una entrevista a los postulantes para aclarar conceptos sobre la Carrera y sus alternativas. En este punto es posible vislumbrar la réplica de un modelo de Teatro Universitario, puesto que se tomaban los mismos parámetros de medición del Teatro Experimental de la Universidad de Chile.

El cuerpo académico de la Escuela de Teatro durante 1971 estuvo compuesto por Juan Guzmán Améstica, quien tuvo a su cargo las cátedras Seminario de Siglo de Oro y Actuación y quien, además, era el director de la Escuela; el poeta Eugenio Matus, quien impartió la asignatura Evolución Comparada de la Literatura y el Arte; el literato y músico Enrique Valdés, como profesor de Guitarra I; la socióloga Liliana Larrañaga, docente de Sociología I; y Jaime Silva, a cargo de Actuación y del Seminario de Realismo.

A fines de agosto de ese año, se presentó el preestreno del examen correspondiente al tercer semestre de la Escuela de Monitores. Las obras escogidas fueron dos entremeses de Miguel de Cervantes, EI Viejo Celoso y La Cueva de Salamanca, las que recibieron una favorable crítica. En cuanto al lugar físico dónde se mostraron estas obras de teatro, al igual que la mayoría de las presentaciones de la

${ }^{3}$ Los directores de la Escuela de Teatro fueron: Juan Guzmán Améstica, 1971-1973; Jaime Silva, 1973-1975; y Lina Ladrón de Guevara, 1975-1976. 
Escuela de Teatro, fue en una cineteca llamada Cine Club, situada al interior del Campus Isla Teja y que se mantiene vigente hasta el día de hoy. La ciudad de Valdivia contaba con el Teatro Cervantes, propiedad de la municipalidad, pero el espacio era utililizado para otros fines (Ossa, 2011).

Al transcurrir los semestres de la Carrera, se fue potenciando la labor que desarrollaba el Área de Extensión. La estrecha relación que mantenían con su entorno les permitió a los estudiantes vincularse directamente con la realidad de su localidad, diferenciándose la Escuela en este aspecto de otros modelos de formación teatral existentes en el resto del país. Así, de acuerdo con Consuelo Holzapfel:

[...] igual había un espacio para mostrar un teatro mejor implementado y más formal, pero la mejor experiencia de Extensión de la Escuela era en lugares impensados: comedores de colegio, un gimnasio, una plaza. Llevamos teatro al campo, a los potreros, a un galpón. Teníamos que sacar la paja para poder instalar la escenografía; imagínense que les tenga que iluminar un tractor porque no hay luz, que tengan que hacer antorchas con paños de las viejas del lugar y untarlas en parafina para poder ver (Holzapfel, 2011).

En las giras que llevaron a cabo los estudiantes de la Escuela de Monitores, se mostraron obras de teatro a los lugareños del interior de Paillaco y de otras pequeñas localidades rurales aledañas a la ciudad, en donde la mayoría de esas personas nunca habían visto una obra de teatro. De esta forma se generaba público y, además, se cumplía con la necesidad de vincular al teatro a personas no relacionadas con la incipiente actividad teatral valdiviana. Esta particular labor se convertiría en el sello de la Escuela.

El Departamento de Teatro era el encargado de recibir las peticiones de los lugares interesados en ver las obras montadas por la Escuela. Asimismo, estaba encargado de facilitar los buses de los que disponía la Universidad, con una capacidad para 45 personas, en donde transportaban al elenco y los académicos responsables. Estas giras, recuerda Margarita Poseck, eran financiadas por la misma institución:

En esa época la universidad tenía una micro horrorosa, que se llamaba La Pingüina. Nos íbamos en la Pingüina y nos demorábamos dos días en llegar a Chiloé. Se hacía mucha itinerancia. Había convenios; por ejemplo, un convenio con el fundo Tucunagüe de la Universidad, al que teníamos que ir todos los meses de octubre y noviembre a hacer funciones a la gente del campo, había mucho que hacer, o sea, nosotros no parábamos [...] (Poseck, 2011).

Cabe mencionar que estos buses no sólo fueron utilizados como medio de transporte para los integrantes de la Escuela, sino también para facilitar el acceso al público general, desde el centro de la ciudad al Cine Club ubicado en Isla Teja. 
Durante 1972 se realizaron las "Jornadas de Teatro Chileno", las que consistían en dos días de presentaciones de cuentos folklóricos. El responsable de la introducción de los cuentos fue el docente Jaime Silva y el reparto estaba conformado por Consuelo Holzapfel en De gancho en gancho y Adriana Becerra en La Tenquita y Los Cuentos del Diablo. El segundo día se presentó la obra Rigoberto, de Armando Moock, cuya introducción estuvo a cargo del ensayista y crítico literario chileno Grínor Rojo (El Correo de Valdivia, 1972a: 8). Finalmente, en el mes de diciembre de ese año, la Escuela de Teatro presentó una obra de Daniel Barros Grez dirigida por Juan Guzmán Améstica (El Correo de Valdivia, 1972b: 8).

Al año siguiente, el 13 de marzo de 1973, se publicó el llamado para participar en la Escuela de Monitores en el periódico local El Correo de Valdivia. Cabe destacar que este medio de comunicación cumplía un rol fundamental en las convocatorias y la difusión de las actividades de la Escuela, pues la mayoría de los estudiantes que ingresaban a la Carrera se enteraban principalmente por el aviso en el periódico o por charlas que brindaban los estudiantes a distintas carreras dentro de la misma universidad y en colegios de la zona.

Aún antes de iniciarse el año académico, el Departamento de Teatro de la Universidad Austral preparó dos comedias en un acto para presentarlas en abril ante los alumnos de los cursos del primer nivel. El objetivo era demostrarles que, al pertenecer a cursos superiores, podrían dirigir obras. Éste fue el caso de Roberto Matamala, quien tuvo la posibilidad de dirigir El Oso y La petición de mano, de Antón Chejov, dónde actuaron principalmente los profesores de la Escuela Hernán Poblete, Jaime Silva y Pablo Carrillo, junto a la actriz invitada Lina Ladrón de Guevara (Matamala, 2011). Por su lado, Consuelo Holzapfel tuvo la oportunidad de dirigir a grupos del Colegio Alemán para realizar su práctica profesional.

A principio de ese año, Jaime Silva pasó a ser el Director de la Escuela de Teatro y Juan Guzmán Améstica prosiguió con labores de docencia. Silva se proyectó con la idea de reforzar el área de actuación, de manera de brindar un nuevo campo laboral a los Monitores y que estos tuvieran la posibilidad de especializarse en esa disciplina. Dicho de otro modo, su intención era cambiar el título de la "Escuela de Monitores Teatrales", por el de un "Taller de Teatro profesional" que enfatizara la formación actoral. Respondiendo a esta idea, invitó a trabajar como docente a Lina Ladrón de Guevara, quien se estableció en Valdivia y dictó las cátedras de Expresión Corporal y Actuación. También invitó al compositor Luis Advis, con quien mantenía una relación de amistad de varios años y habían llevado a cabo numerosas presentaciones en Antofagasta y Santiago.

El 11 de septiembre de aquel año 1973, como se sabe, se produjo el golpe de Estado encabezado por Augusto Pinochet Ugarte. El nuevo régimen autoritario 
perpetró diferentes cambios administrativos al interior de las academias de teatro, pese a que, en sus inicios, no poseía ningún plan de acción común respecto a las escuelas de artes escénicas universitarias. En el Teatro Experimental de la Universidad de Chile en Santiago, el grupo teatral se vio afectado por las medidas de las nuevas autoridades, sufriendo la expulsión de varios actores del elenco, mientras que, en la Escuela de Teatro de la sede Valparaíso, se publicaron listas negras de personas que ya no podían continuar en la academia, entre ellas, Juan Barattini Carvelli (Barattini, 2011). En la UACH, en tanto, se realizaron cambios administrativos cuya intención era la de extinguir poco a poco la Escuela de Teatro.

Paradójicamente, mientras el país se encontraba inmerso en un régimen opresor, Jaime Silva veía cómo su carrera profesional iba en ascenso. Entre las múltiples actividades que lideró, cabe destacar un taller de Luthería dentro de la Facultad, donde se enseñaba a reparar y construir violines y guitarras. Además, creó los Viernes Culturales, instancia amparada por la Universidad en la que se turnaban espectáculos de teatro, danza y coro. En este evento se podía contar con compañías de teatro capitalinas que presentaban sus obras al público local. Sin embargo, a pesar de ser muy productiva y beneficiosa para el teatro chileno descentralizado, la tarea que Silva intentó realizar fue bastante mal vista por sus colegas del entorno teatral.

Incluso yo, conversando con Jaime, me enteré que cuando regresó después de muchos años a Santiago él fue prácticamente vetado por sus colegas y le fue muy mal (...) porque se le estigmatizó como pro dictadura y él siempre lo dijo: "yo me hice cargo de Valdivia para salvar la Escuela de Teatro, para que no muriera yo me vine a Chile", pero nadie lo entendió así (Poseck, 2011).

A fines de 1973, la falta de claridad en el reglamento respecto a la obtención del grado académico de Licenciado en Teatro con mención en Actuación, sumado a un hostil contexto para las artes escénicas, clausuró la posibilidad de obtener dicho grado, dejando solamente la opción de obtener un diploma de un "Taller de Teatro". Sin embargo, este título no contaba con una visión propia del fenómeno artístico, sino que pretendía formar a una especie de guía para actividades en educación primaria, según recuerda Margarita Posek:

Es que en realidad cuando la escuela se cerró para el 73, nunca más volvió a ser escuela. En esa época era todo tan raro, la universidad estaba pasando por un momento muy complicado, entonces se hablaba de nosotros por la prensa como Taller de Teatro. Académicamente eso no existía, era un Bachiller en Teatro que duraba tres años. Entonces fue una medida de emergencia formar un Bachiller de Teatro que, una vez que saliera la primera promoción, la promesa era que se nos contrataba para formar una compañía profesional. Entonces, incluso nosotros, ya el último año del bachiller, estábamos contratados. Yo tenía un sueldo en la universidad, que era la nada, como una especie de ayudantía, lo que se conoce hoy día como una laborancia. Fíjense que todos 
esos conceptos no estaban definidos, pero en realidad era un Bachiller en Teatro con mención en Actuación (Poseck, 2011).

La idea inicial de Silva de formar actores, más que monitores teatrales, permitió que la labor de la Escuela pareciera más inofensiva ante la mirada inquisidora de las autoridades militares, las que poco interés tenían en mantener, y mucho menos fomentar, alguna actividad relacionada con las artes escénicas. Para el gobierno de facto, un "Taller de Teatro" era una actividad escénica mucho menos peligrosa y más restringida que la asociada a la formación de Monitores Teatrales. Lamentablemente, este cambio fue el primer paso hacia la clausura de la Escuela de Teatro. El testimonio de Consuelo Holzpafel es claro al respecto:

De lo que yo recuerdo después del 73, del golpe, se hizo cargo como rector de la Universidad un milico... Hasta hace poco que me acordaba del nombre de ese milico porque era casi como un chiste, era un milico bestia, bueno, como lo fueron muchos en esa época. [...] Para frenar a la universidad de estos cabros sublevados, llegó este milico mano dura, sacó gente, cambió, hizo lo que quiso. [...] No lo exterminó de un día para otro, pero le bajó el perfil a todo y esta Escuela de Teatro paso a ser un Taller. Y como a los dos años, ya no quedó nada [...] (Holzapfel, 2011).

Sin duda, fueron momentos muy complejos para las artes escénicas y para quienes trataron de continuar su quehacer bajo este contexto. Por los motivos mencionados, la Escuela de Teatro disminuyó su ritmo de presentaciones, lo que afectó claramente a los grupos. Sin embargo, un hecho fortuito influyó de manera directa en su continuidad:

[...] En el año 74 existía un rector que era de apellido Dupuis [...]. Era uno de estos delegados milicos, militar, y a él le encantaba el teatro, tanto así que este señor nos ponía muy nerviosas porque se iba meter a los camarines, o sea, llegaba y se sentaba a mirar cómo nos maquillábamos. Lo fantástico era que, en el teatro, nos daba todo lo que nosotros quisiéramos; nos facilitaba las micros y financiamientos para las giras. Incluso a nosotros nos pasaron toda la planta de arriba del conservatorio, yo me acuerdo porque estaba a cargo del vestuario, y teníamos de todo (Poseck, 2011).

De esta manera, y supeditado a las preferencias de los militares designados en sus cargos, se continuó el trabajo del Taller de Teatro durante tres años más, pese a lo cual surgieron nuevas limitaciones debido a una serie de factores administrativos y, también, políticos. Notablemente, tuvieron que atenerse a la censura a la hora de elegir su nuevo repertorio teatral, lo cual Jaime Silva tuvo muy en cuenta para no poner riesgo la sobrevivencia de la Escuela (Poseck, 2011). 


\section{Breve análisis de la malla curricular de la Escuela de Teatro $\mathrm{UACH}^{4}$}

En la malla curricular de 1970, es posible observar un intento de visión integrada de las Artes Escénicas, lo cual se refleja en la presencia de las siguientes cátedras: Guitarra I, impartida por el poeta Enrique Valdés; Evolución Comparada de la Literatura y el Arte, impartida por Eugenio Matus; y Sociología, dictada por Liliana Larrañaga, quién también impartía clases en la Facultad de Filosofía y Letras dentro de la misma casa de estudios. La particular orientación académica de la Escuela de la UACH en este primer período hacía que sobresaliera por algo más que su ubicación en la zona sur del país. En efecto, este enfoque en la formación de Monitores no sólo la diferenciaba del resto de los Teatros Universitarios, sino que, hasta nuestros días, no se ha vuelto a repetir con las mismas características. Aún así, se estipulaba lo siguiente en el Reglamento de la Escuela:

Aquellos monitores que hayan demostrado especiales condiciones para la actuación o dirección teatrales podrán solicitar de la Escuela que se le programe un curriculum complementario para obtener el grado académico de Licenciado en Teatro con mención en Actuación y/o Dirección. [...] La realización de estas especialidades estarán sujetas a las posibilidades reales de la Escuela (UACH, 1972: 3).

Esta cláusula dejaba en evidencia una ambigüedad en lo que se refería al criterio de selección de los alumnos que podían llegar a obtener tal grado, puesto que no estaban explicitados, en el mismo reglamento, ni la malla curricular, ni el procedimiento correspondiente. Por ello, no es posible confirmar ni los parámetros utilizados, ni si efectivamente existían programas curriculares especiales para estos casos, ya que tampoco existe registro de que efectivamente alguien se haya titulado bajo estas menciones.

La malla curricular del segundo período, además de no incluir los ramos teóricos que distinguían a la Escuela de la UACH del resto de las Escuelas de Teatro, proponía un enfoque alejado del ámbito social. A partir de 1974, el objetivo de la Escuela fue el de formar actores, por lo que su plan de estudios dio un giro hacia lo práctico, volviendo más técnica la enseñanza y disminuyendo en un año la malla curricular. Al dejar de ser una Escuela de Monitores Teatrales, se restringió el campo laboral de los futuros artistas escénicos, se eliminó el impacto social que pretendía tener la Carrera y, además, se perdió su peculiaridad frente a las demás escuelas del país.

De todas formas, este segundo período terminó en 1976, antes de finalizar el año académico, por lo que nadie egresó de la Carrera bajo esta nueva modalidad.

${ }^{4}$ Ver Anexo sobre Mallas Curriculares. 
Ese mismo año, por determinaciones políticas ajenas a su quehacer, se cerró definitivamente la Escuela de Teatro de la Universidad Austral de Chile.

\section{Segundo periodo, 1974-1976}

Durante los años 1974 a 1975, Jaime Silva pasó a ser Decano de la Facultad de Bellas Artes, delegando la Dirección de la Escuela de Teatro a la docente Lina Ladrón de Guevara. En ese entonces, el Taller de Teatro tenía dos tareas fundamentales: la primera de ellas era formar actores y técnicos teatrales; la segunda, conquistar al público de la ciudad para darle a conocer importantes obras de la dramaturgia universal, cooperando así con el desarrollo cultural de la región. Sus giras durante ese año abarcaron desde Chiloé hasta Concepción, contando con miles de espectadores a lo largo del sur chileno.

La labor realizada por el Taller de Teatro tendría como punto cúlmine, ese mismo año, el montaje de la obra El Oratorio Escénico 1850, original de Luis Advis y Jaime Silva. Creada en cinco meses con el objetivo de celebrar los 25 años de la Universidad, su música fue compuesta por Advis y su dramaturgia por Silva, quien se basó en testimonios orales y relatos sobre la historia de Valdivia. Explicaba este autor: "el trabajo fue duro, pero valió la pena. Estuvimos trabajando desde febrero y en julio ya teníamos 'El Oratorio' listo para ensayar" (El Correo de Valdivia, $\mathrm{SF})$.

En la obra trabajaron más de cien personas, pues la componían los dos coros de la Universidad Austral y el elenco estable de la Escuela, además de Malú Gatica y Alberto Mery, quienes eran los narradores de la obra. Debido a que el Cine Club no tenía la capacidad para albergar a un elenco de tal magnitud, El Oratorio fue estrenado en el Teatro Cervantes de Valdivia.

El montaje tuvo una fuerte repercusión pública. No existían precedentes de una pieza teatral sobre la llegada de los colonos a esa ciudad y esta temática histórica regional hizo que la puesta en escena pasara a ser un exponente del teatro que se venía desarrollando en la Facultad de Bellas Artes. Paradójicamente, todo este ascenso se producía en el contexto de la dictadura y bajo la protección oficialista. En cuanto a su temática, Margarita Poseck agregó:

[...] Era una obra donde se contó cómo los colonos alemanes hicieron nacer y renacer esta tierra, incluso el texto lo dice: "ésta tierra renacerá, la tierra de los vivos". Es una apología. Ahora, igual hubo muchos alemanes que se sintieron ofendidos porque no estaban todos, mencionaban a algunos, típico, ¿no? Pero en realidad es una apología a la colonización alemana de todo lo que hizo, todo lo que significó para esta ciudad su llegada (Poseck, 2011).

Se efectuaron giras a la zona central del país, con funciones en los teatros municipales de Viña del Mar, Valparaíso y Santiago, y también en la zona Sur, en las 
ciudades de Concepción, Temuco y Puerto Montt. Por ello, con este montaje, la Escuela de Teatro de la UACH consolidó su trabajo escénico, alcanzando reconocimiento a nivel nacional.

En el año 1975, se estrenó, el 11 de abril, una obra infantil en la Secretaría de la Mujer de Valdivia, titulada Trauco Enamorado. Dicha presentación sirvió para dar a conocer a la Directora del Taller, Lina Ladrón de Guevara, ante los intendentes, el General Soler, de Puerto Montt, y el General Fernando Paredes, de Valdivia. Este tipo de iniciativas demostraban la necesidad de mantener una relación cordial entre la Escuela de Teatro y los militares que gobernaban en la época, con el fin de mantener la actividad teatral universitaria. Aunque, para lograrlo, se debía abandonar toda crítica social, la que, obviamente, no era permitida en el régimen autoritario imperante.

La segunda muestra estrenada ese año, fue Amor x 2, el 12 de junio, que incluía dos piezas de distintos autores. Una de ellas era Cheché, de Luigui Pirandello, en la que participaban Bruno Rodríguez, Juan Ossa y Eugenia Poseck. La segunda pieza, titulada La Carroza del Santo Sacramento, del escritor francés Próspero Merimée, fue dirigida por Jaime Silva. Amor x 2 se presentó en el Cine Club del campus Isla Teja, recibiendo una crítica favorable de parte del periódico local. A continuación, el 5 de septiembre, se pre-estrenó la obra Como en Santiago, escrita por Daniel Barros Grez. Esta muestra primero tuvo lugar en Santiago, en el Rotary Club, y, tres días después, se estrenó oficialmente en el Cine Club de la $\mathrm{UACH}$.

Durante el mismo año 1975 se llevaron a cabo diversas giras en el centro y sur del país, con el propósito de dar a conocer el trabajo artístico de la Escuela. Entre los lugares visitados estuvieron Ancud, Castro, Achao, Osorno, Temuco Pilmaiquén, Pullinque y San José, además de Santiago y Valparaíso. Asimismo, se realizaron funciones benéficas para el Colegio Alemán de Valdivia, el Liceo de Hombres, el Liceo de Niñas, el Windsor School, el Liceo Comercial, el Rotary Club de Valdivia, la Secretaría de la Mujer y para diversos Centros de padres y Centros de alumnos. Paralelamente, se llevaron a cabo temporadas de carácter educativo con el apoyo de diversos establecimientos educacionales.

En pleno apogeo de la dictadura militar, como es sabido, se persiguió a las personas simpatizantes de los partidos asociados a la izquierda chilena, entre los cuales se encontraba Luis Advis. Músico y filósofo, compuso múltiples piezas que luego fueron interpretadas por Quilapayún e Inti-Illimani, entre otros, siendo la Cantata de Santa María de Iquique, emblema de de los militantes de la Unidad Popular, una de sus composciones más destacadas. En 1975, cuando fueron en su búsqueda, él se cobijó al amparo de la Universidad, motivo por el cual pudo 
quedarse en Chile (Poseck, 2011). Por otra parte, pese al apoyo moral que representaba el rector designado Dupuis, por su afición al teatro, los recursos económicos fueron reduciéndose con el paso de los meses, por lo que el futuro de la Escuela continuó pendiendo de un hilo.

A fines de 1975, luego de una reunión a la que asistieron los profesores de castellano de todos los establecimientos de educación media y los profesores de la Escuela de Teatro de la $\mathrm{UACH}$, se reconoció la importancia del teatro como vehículo de motivación de las actividades estudiantiles, puesto que los programas de educación media incluían el estudio del teatro en sus diversas manifestaciones y periodos. De este modo, se estableció la más amplia cooperación entre el teatro universitario y los establecimientos medios de la región, con el propósito de que todos los alumnos tuviesen la oportunidad de ser espectadores de los montajes. De allí que en la preparación de todos los estrenos del Taller de Teatro se consideraran diversas presentaciones especiales para estudiantes, quienes, además, podían asistir a ellas de forma gratuita.

En el mes de marzo de 1976, mientras Lina Ladrón de Guevara seguía siendo la Directora del Taller de Teatro, los militares a cargo de la Universidad Austral dictaron un decreto que determinaba la estructura interna y los objetivos de la Facultad de Bellas Artes. Este decreto fue firmado por el Rector Delegado Gustavo Dupuis Pinillos y por el Secretario General Hernán Poblete Varas. A partir de ese momento, se fue asfixiando la continuidad del Taller de Teatro, hasta que, en noviembre de ese año, la Escuela de Teatro de la UACH se cerró definitivamente.

Debido a que los entrevistados entregaron distintas versiones respecto de los motivos concretos del cierre de la Escuela de Teatro, intentamos aunarlas para generar una historia común. Una historia que hemos pretendido recomponer a partir de diversas anécdotas, testimonios y puntos de vista diferentes, pero en un contexto preciso y, sin duda, determinante.

Según el testimonio de Margarita Poseck, un día sábado de 1976, tras una de las presentaciones del reestreno de la obra Oratorio Escénico 1850, se llevó a cabo una celebración entre los actores, en la casa de uno de ellos. En la mitad de la celebración, llegó el Rector Dupuis con su esposa Alda Villalta. Esto generó una atmósfera más bien densa, ya que todos estaban extrañados por esta repentina aparición. Además, en ese mismo momento, dos personas no identificadas tuvieron que ser trasladadas al hospital de Valdivia, a raíz de una golpiza que tuvo lugar en el transcurso de la fiesta. Este hecho fue de conocimiento público, contribuyendo a desprestigiar al Taller de Teatro al punto de menospreciarse la trayectoria que había conseguido con bastante esmero. Tras este incidente, se provocó la división del elenco de la obra: quienes integraban el coro, que no participaron en la fiesta, consideraron reprochable la actitud de los actores; los actores, por su 
parte, estimaban que lo ocurrido no tenía el peso suficiente como para afectar el desempeño y la existencia del Taller de Teatro.

Pese a que la situación era insostenible, el elenco de El Oratorio Escénico 1850 aún tenía una función pendiente. Por este motivo, Jaime Silva decidió llevar a cabo de todas maneras el montaje, debiendo remplazar actores por personas externas a la Escuela, pero que poseían cierta noción respecto al orden de los cuadros y escenas. Recuerda Margarita Poseck:

Gabriel Rojo, que era la pareja de Jaime cuando llegó acá (poeta y todo el cuento), nunca fue actor. Con él trabajábamos Artaud y todas esas cosas, pero nunca perteneció. Al primero que subimos al escenario fue a Gabriel. Estaba lleno de gente que no tenía nada que ver, pero que habían visto todo. Los pololos de algunas actrices, para arriba, porque habían estado en el proceso y sabían lo que había que hacer. Fue una cosa demencial, todo porque esa función estaba vendida. Finalmente se hizo la función y eso provocó el cierre definitivo (Poseck, 2011).

La función fue un día sábado y el lunes siguiente se encontraron con un gran candado en la puerta, por lo que ni siquiera pudieron recuperar sus vestuarios, su escenografía ni sus pertenencias personales.

Frente a este desfavorable panorama, Jaime Silva tomó la determinación de regresar a Santiago. Antes de partir de la ciudad de Valdivia, delegó el Decanato de la Facultad a Hernán Poblete Varas, quien fue Decano de Bellas Artes en el período 1976-1977, y dejó como Secretario General a Félix Urcullu Molina. Por otro lado, en diciembre de 1976, el puesto de Rector Delegado lo ocupó el militar Pedro Palacios Cameron, a quien no le agradaba la disciplina teatral. En ese momento, las posibilidades de continuar el Taller se esfumaron por completo (Poseck, 2011).

Otro factor presente en las distintas versiones acerca del cierre definitivo del Departamento de Teatro y su Escuela fue el económico, planteado en especial por Juan Ossa. La reducción de fondos se hizo inminente tanto en la Facultad de Bellas Artes como en toda la UACH, por lo que el escenario fue cada vez más adverso. Para el testigo, otro posible motivo es que la disciplina teatral siempre ha sido muy peligrosa para los regímenes totalitarios (Ossa, 2011). Evidentemente, lo que vuelve peligrosa la experiencia teatral es en primer lugar la posibilidad de convivio sostenido entre espectadores, actores y técnicos, lo que contradecía la prohibición de reunión. En este contexto, era necesario erradicar todo tipo de manifestación artística que vulnerara el sistema hermético que se quería implementar. Además, debía suprimirse todo mecanismo que fuera un posible portador de mensajes políticos. 
Por lo anterior, el desenlace de la Escuela de Teatro de la UACH fue inevitable y todos los esfuerzos que se hicieron por mantener en pie la actividad teatral universitaria valdiviana terminaron fracasando. Frente a un gobierno dictatorial que cercenó el arte escénico en cada oportunidad que se le presentó, especialmente estando en una ciudad tan aislada geográficamente, ganar esta lucha fue simplemente imposible.

\section{Conclusiones}

Basándonos en la investigación realizada, podemos afirmar que el proyecto de la Escuela de Teatro de la Universidad Austral tenía rasgos originales tanto en su fundación como en su desarrollo posterior en la ciudad de Valdivia, viéndose truncada su historia como consecuencia de la dictadura que vivió Chile a partir del año 1973. Pese a su corta trayectoria, hemos podido identificar ciertas diferencias y similitudes respecto a las otras escuelas de formación teatral universitaria existentes $y$, en especial, frente al modelo generado en la capital.

En efecto, este modelo de formación teatral universitaria implementado en la Universidad de Chile fue replicado tanto en Santiago como en regiones, pero sufriendo pequeñas modificaciones que respondían a las necesidades y condiciones de cada localidad. Por otro lado, la Reforma Universitaria también sirvió para promover la génesis de Escuelas de Teatro fuera de Santiago, pues, al brindarle un mayor énfasis al ámbito educacional, provocó una popularización del acceso a la cultura y al arte como una herramienta de transformación social. El objetivo era la descentralización de la actividad teatral, para, de esta forma, enriquecer la disciplina a nivel nacional con el intercambio de geografías y costumbres.

Ahora bien, en el caso particular de la UACH, la Escuela comenzó teniendo un enfoque distinto en su primera etapa, del año 1970 al 1973, ya que buscaba la formación de Monitores Teatrales. Por ello, puede decirse que, si bien no se distinguió del modelo establecido por la Universidad de Chile, sí presentó diferencias en cuanto al plan de estudios y, por ende, la orientación académica. En el período siguiente, posterior al golpe de Estado, tuvo lugar un cambio significativo en la dirección de la Carrera de la UACH, de manera que, a partir de 1974, en vez de formar Monitores Teatrales, comenzó a formar Actores. Los beneficios que se habían obtenido como consecuencia de la Reforma Universitaria y que luego se desarrollaron y potenciaron en el Gobierno de la Unidad Popular de Salvador Allende fueron eliminados, generándose durante la Dictadura un proceso de restricción y pauperización cultural.

Pueden observarse otras similitudes entre la Escuela de Teatro de la UACH y las demás escuelas de formación teatral universitaria. Por ejemplo, antes de la aparición del teatro universitario en Valdivia, ya existía un fuerte movimiento de teatro aficionado en la ciudad, lo que luego se vio reflejado en las relaciones de la 
Escuela de la UACH con el contexto local. Además, la fundación de esta escuela se debió a la acción de Rubén Sotoconil, quien era un actor clave en el ámbito teatral, como lo fueron en otros lugares distintos individuos, grupos y asociaciones (el caso de ATEVA y Marcos Portnoy en Valparaíso). De hecho, los Teatros Universitarios se potenciaron y consolidaron gracias a la inquietud de grandes maestros del teatro que se encontraban ligados a la pedagogía, varios de cuyos nombres se repitieron en las diversas iniciativas de formación teatral y compañías teatrales universitarias: es el caso del mismo Rubén Sotoconil en la Universidad de Chile y la Universidad Austral; de Pedro de la Barra en la Universidad de Chile, la Universidad Católica y la Universidad de Chile sedes Antofagasta y Valparaíso; y de Jaime Silva en la Universidad de Chile sedes Santiago y Antofagasta, así como en la Universidad Austral, por nombrar sólo algunos.

Otro rasgo de la Escuela de Teatro de la UACH similar al de otras escuelas de teatro universitario es el impacto que tuvo sobre ella el contexto de movilización social y posterior represión política. Con la Reforma Universitaria, el teatro pasó a ser una herramienta política utilizada tanto para llegar a los sectores populares como en campañas gubernamentales, lo que se tradujo en la UACH en el mencionado proyecto académico de formación de Monitores Teatrales. Un par de años después, la misma escuela se vio afectada por el golpe de Estado y la dictadura que vivió Chile hasta el año 1990, poniéndose fin a su nuevo enfoque social y también, en definitiva, a su propio proyecto universitario, al igual que en el caso de Valparaíso.

Existe aquí una diferencia regional, pues, mientras que las escuelas de la Universidad de Chile y la Universidad Católica de Chile siguieron funcionando hasta nuestros días, la Carrera de Teatro de la Universidad de Chile sede Valparaíso fue cerrada y, tras más de treinta años, renació escindida en dos instituciones estatales: la Universidad de Playa Ancha y la Universidad de Valparaíso. En la actualidad, ambas imparten profesionalmente la formación de actores y han logrado consolidarse en sus respectivas instituciones y regiones, pero en Valdivia no se ha vuelto a implementar la formación teatral universitaria.

Debido a ello, podemos concluir que este tipo de formación sólo ha logrado subsistir hoy en la zona central del país. Si bien han surgido diversas iniciativas para abrir carreras de teatro en lugares alejados de la zona central, han debido cerrarse, por tratarse de iniciativas privadas sometidas a las leyes de mercado. En el caso puntual de Valdivia, a pesar de haber mejorado su acceso geográfico con la implementación de carreteras y la modernización de infraestructura, su densidad de población es muy baja, lo que vuelve poco rentable abrir una carrera de teatro según el sistema universitario vigente. La Reforma Educacional de 1982 aplicada durante el Régimen Militar encasilla los estudios de arte como un "mal 
negocio", puesto que no conducen a una economía sustentable. Esta visión neoliberal de la educación es promovida por estudios de mercado y rankings que asocian el éxito de un profesional con sus logros económicos.

Lo anterior hace evidente que, de no existir una decisión por parte del Estado de generar un polo de desarrollo artístico en regiones, la formación teatral universitaria no es viable en Valdivia. Lamentablemente, el modelo implantado en Dictadura, que significó asimismo la implementación del mecanismo de los fondos públicos concursables en las postrimerías del régimen, fue adoptado por los gobiernos posteriores. Éstos, en efecto, se han limitado a profundizar estos fondos, sin establecer políticas culturales de largo plazo que superen los períodos presidenciales y que signifiquen cambios sustantivos en zonas del país alejadas de la capital. Los desarrollos artísticos reales están asociados a políticas gubernamentales que pueden, si así lo deciden, generar un desarrollo descentralizado e inclusivo en regiones distantes de la capital. Por ello se vuelve fundamental documentar y visibilizar el hecho de que, en otra época, hubo políticas hacia el teatro y las artes distintas a las actuales. Ha sido nuestra intención al rescatar parte de la memoria teatral chilena, en un país que ha desarrollado políticas amnésicas en el transcurso de su historia.

\section{Referencias}

Almonacid Zapata, Fabián (2004). Historia de la Universidad Austral de Chile (1954-2003). Su historia en el contexto universitario nacional. Valdivia: Ediciones Universidad Austral de Chile.

El Correo de Valdivia (1972a). Función de la Escuela de Teatro de la Facultad de Bellas Artes. Valdivia: 18 de diciembre 1972.

(1972b). Jornadas de Teatro Chileno . Valdivia: 13 de diciembre 1972.

Hurtado, María de la Luz (2003). (CD-Rom) Chile, 1941-2002: Los Teatros Universitarios en Escena- Historia crítica y memoria (audio)visual. Santiago de Chile: Programa de Investigación y Archivos de la Escena Teatral - Escuela de Teatro de la Facultad de Artes de la Pontificia Universidad Católica de Chile.

(2010). Chile Actúa. Teatro Chileno. Tiempos de Gloria (19491969) Desde la fotografía de René Combeau. Santiago de Chile: Programa de investigación y archivos de la escena teatral UC - Facultad de Artes - Pontificia Universidad Católica de Chile.

Hurtado, María de la Luz \& Barría, Mauricio (2010). Antología un siglo de Dramaturgia Chilena, tomo II 1950- 1973. Santiago de Chile: Publicaciones Comisión Bicentenario Chile 2010.

Moulian, Tomás (1997). Chile Actual. Anatomía de un mito. Santiago de Chile: LOM Ediciones. 
Ochsenius, Carlos (1982). El Estado en la escena. Teatros Universitarios de Santiago: 19401973. Santiago de Chile: Pontificia Universidad Católica de Chile.

Pradenas, Luis (2006). Teatro en Chile, huellas y trayectorias. Siglo XVI-XX. Santiago de Chile: LOM.

Revista Apuntes (2003). Publicación de la Escuela de Teatro. Pontificia Universidad Católica de Chile. Apuntes de Teatro, (123-124).

San Martín, Angélica; Sotomayor, Sibila (2012) Una escena truncada: historia de la Escuela de Teatro de la Universidad Austral de Chile (1970-1976). Memoria de grado dirigida por Verónica Sentis Herrmann. Valparaíso: Escuela de Teatro, UV.

Sotoconil, Rubén (1970). Carta dirigida a Mireya Moreno, 22 de abril 1970. Valdivia.

(1991). A 50 años de la fundación del Teatro Experimental de la Universidad de Chile. Apuntes de Teatro (102).

Universidad Austral de Chile (1972). Reglamento Escuela de Teatro, Facultad de Bellas Artes. Valdivia.

Zegers, María Teresa (1999). 25 Años de Teatro en Chile. Santiago de Chile: Ministerio de Educación.

\section{Entrevistas}

Barattini, Juan (2011). Entrevista 20 Abril 2011, Viña del Mar.

Holzapfel, Consuelo (2011). Entrevista 27 de abril 2011, Viña del Mar.

Matamala, Roberto (2011). Cuestionario vía correo electrónico, Valdivia.

Ossa, Juan (2011). Entrevista 4 de mayo 2011, Valdivia.

Poseck, Margarita (2011). Entrevista 03 de mayo 2011, Valdivia. 


\section{Anexo}

\begin{tabular}{|c|c|c|c|c|}
\hline \multicolumn{5}{|c|}{ Escuela de Teatro Universidad Austral - Malla curricular } \\
\hline \multicolumn{2}{|c|}{$1970-1973$ ( 8 semestres) } & \multicolumn{3}{|c|}{ 1974-1976 (6 semestres) } \\
\hline $\begin{array}{c}\text { Asignaturas y Actividades } \\
\text { Curriculares }\end{array}$ & Año & $\begin{array}{c}\text { Asignaturas y Actividades } \\
\text { Curriculares }\end{array}$ & Semestre & Año \\
\hline Actuación & 1 & Actuación I & 1 & 1 \\
\hline Movimiento & 1 & Expresión Corporal I & 1 & 1 \\
\hline Maquillaje & 1 & Maquillaje I & 1 & 1 \\
\hline Voz & 1 & Esgrima I & 1 & 1 \\
\hline Historia del Teatro & 1 & Prácticas de Actuación I & 1 & 1 \\
\hline Actuación & 2 & Historia del Teatro I & 1 & 1 \\
\hline Movimiento & 2 & Educación Hablada I & 1 & 1 \\
\hline Seminario Siglo de Oro & 2 & Actuación II & 2 & 1 \\
\hline Guitarra I & 2 & Expresión Corporal II & 2 & 1 \\
\hline Sociología & 2 & Maquillaje II & 2 & 1 \\
\hline Realismo Épico I & 2 & Esgrima II & 2 & 1 \\
\hline Realismo Épico II & 2 & Prácticas de Actuación II & 2 & 1 \\
\hline Seminario Realismo I & 2 & Historia del Teatro II & 2 & 1 \\
\hline Seminario Realismo II & 2 & Expresión Oral & 2 & 1 \\
\hline $\begin{array}{l}\text { Evolución comparada } \\
\text { de la Literatura y el Arte }\end{array}$ & 2 & $\begin{array}{l}\text { Gimnasia general y Des- } \\
\text { treza en colchoneta I }\end{array}$ & 2 & 1 \\
\hline Actuación & 3 & Actuación III & 3 & 2 \\
\hline Producción de la Voz & 3 & Expresión Corporal III & 3 & 2 \\
\hline $\begin{array}{c}\text { Didáctica Teatro Infan- } \\
\text { til }\end{array}$ & 3 & $\begin{array}{l}\text { Prácticas de Actuación } \\
\text { III }\end{array}$ & 3 & 2 \\
\hline Dinámica de Grupo & 3 & Historia del Teatro III & 3 & 2 \\
\hline Historia del Teatro I & 3 & Educación Hablada II & 3 & 2 \\
\hline $\begin{array}{l}\text { Historia del Teatro Chi- } \\
\text { leno }\end{array}$ & 3 & $\begin{array}{l}\text { Gimnasia general y Des- } \\
\text { treza en colchoneta II }\end{array}$ & 3 & 2 \\
\hline
\end{tabular}


Panambí n. 2 Valparaíso jun. 2016 ISSN 0719-630X. 181-203.

\begin{tabular}{|c|c|c|c|c|}
\hline $\begin{array}{l}\text { Teatro Latinoameri- } \\
\text { cano }\end{array}$ & 3 & Actuación IV & 4 & 2 \\
\hline Práctica Dirección I & 4 & Expresión Corporal IV & 4 & 2 \\
\hline Práctica Dirección II & 4 & $\begin{array}{c}\text { Prácticas de Actuación } \\
\text { IV }\end{array}$ & 4 & 2 \\
\hline Voz II & 4 & Historia del Teatro IV & 4 & 2 \\
\hline Historia del Teatro II & 4 & Dirección I & 5 & 3 \\
\hline Teatro Chileno & 4 & Producción & 5 & 3 \\
\hline $\begin{array}{l}\text { Teatro Latinoameri- } \\
\text { cano }\end{array}$ & 4 & $\begin{array}{c}\text { Técnica Literaria del } \\
\text { Drama }\end{array}$ & 5 & 3 \\
\hline \multirow[t]{3}{*}{$\begin{array}{c}\text { Seminario de Aspectos } \\
\text { Técnicos }\end{array}$} & 4 & Dirección II & 6 & 3 \\
\hline & & Seminario de Actuación & 6 & 3 \\
\hline & & $\begin{array}{l}\text { Seminario de Teatro In- } \\
\text { fantil }\end{array}$ & 6 & 3 \\
\hline
\end{tabular}

